\title{
BUI: BROWSER USER INTERFACE AND EXTENSIONS WITH CLOUD STORAGE
}

\author{
Sri Venkatesh S. \\ Panimalar Engineering College, Chennai, India \\ Email: ssvenkatesh6666@gmail.com
}

\begin{abstract}
$\mathrm{BUI}$ : Browser User Interface. BUI is a technology which unifies the desktop environment inside the browser. Implementing such an interface will radically alter the desktop environment. First and foremost, the concept of a separate browser running on a desktop is eliminated. In fact, the browser will be the desktop. The need for having browser as a separate application running on the desktop is eliminated and the browser is made the desktop itself. Such a technology will provide a better access to the internet and better management of work on and off-line. The Browser User Interface will be easier to implement and provides easy use to all its users. This technology basks on the growth of the browsers. The BUl based operating system is perfect for organizations which involves cloud computing for storage. BUI eradicates the explicit connection to the internet and automatically connects to the net while the OS program is loaded. BUI marks the future of the operating systems using browser as desktop interface.
\end{abstract}

Keywords- BUI, Dropbox, Mailbox, STaaS, SECaaS, Skydrive, Cloud Computing, Java, AJAX, .NET, ActiveX, GUI, CLI, OS.

\section{INTRODUCTION}

The user interface is the space where the interaction between humans and machines occur [1]. The goal of the user interface is to facilitate effective operation and control over the machine. User interface includes hardware and software components [2]. The user interface is the part of a computer and its software that people can see, hear, touch, talk to, or otherwise understand or direct. The user interface has essentially two components: input and output. Input is how a person communicates his or her needs or desires to the computer. Some common input components are the keyboard, mouse, trackball, one's finger (for touch-sensitive screens or pads), and one's voice (for spoken instructions). Output is how the computer conveys the results of its computations and requirements to the user [3].

Generally, the goal of human-machine interaction engineering is to produce a user interface which makes it easy, efficient, and enjoyable to operate a machine in the way which produces the desired result. This generally means that the operator needs to provide minimal input to achieve the desired output, and also that the machine minimizes undesired outputs to the human.

Usability is mainly a characteristic of the user interface, but is also associated with the functionalities of the product and the process to design it. It describes how well a product can be used for its intended purpose by its target users with efficiency, effectiveness, and satisfaction, also taking into account the requirements from its context of use. A property of a good user interface is consistency. Good user interface design is about getting a user to have a consistent set of expectations, and then meeting those expectations [3].

\section{TYPES OF USER INTERFACE AND TRANSITION TO BUI}

The most common types of user interface include Command line interfaces and Graphical user interfaces. A command-line interface (CLI) is a means of interaction with a computer program where the user (or client) issues commands to the program in the form of successive lines of text or command lines. In this type of interface, the user provides the input by typing a command string with the computer keyboard and the system provides output by printing text on the computer monitor. CLI is widely used by programmers and system administrators in engineering and scientific environments and by technically advanced personal computer users [4].

A graphical user interface (GUI) is a type of user interface that allows users to interact with electronic devices using images rather than text commands. GUI accepts input via devices such as computer keyboard 
and mouse and provides articulated graphical output on the computer monitor [5].

Most time spent on the computer by an individual is actually spent on the internet. Based on this fact the idea of Browser based user interface is brought up. For users who needs fast access to their online services BUI will be the best choice. Web connections will become more fast and browsers so powerful that most of the programs that currently run on PCs will be replaced by online applications. This would eliminate the need to install, upgrade and backup software.

\section{TECHNOLOGICAL REQUIREMENTS}

The basic requirement in technologies for BUI includes HTML, CSS and a scripting language. The choice of the scripting language is the key in BUI development. The conflict arises between VBScript and JavaScript. The solution for this would be mostly JavaScript, since it is supported by most browsers. In case of a BUI development by Microsoft, there is a possibility of using VBScript.

Apart from these various other technologies such as Java, AJAX, .NET are also required for the development of BUI. Among these, AJAX would be the most prominent technological requirement, because BUI requires constantly updating the desktop without refreshing it. Browser based user interface also requires changes in the hardware components such as memory. BUI also requires ActiveX scripting or Active scripting for Windows to implement component-based scripting support.

\section{STORAGE ON CLOUDS}

The storage for $\mathrm{BUI}$ can either be in secondary storage devices or can be achieved by cloud computing. By having the browser as the desktop, there is no need for connecting to the network explicitly. Browser based user interface automatically connects to the internet once the operating system boots up. The automatic triggering is done by event driven modelling. A software is embedded inside the operating system helps us to achieve such an action.

The browser based user interface is well-suited for organizations. Many organizations use cloud computing for storage purposes. With the $\mathrm{BUI}$ it is easy to access the stored data from the clouds. This is achieved through Storage as a Service (STaaS) and Security as a Service (SECaaS) business models [6].
Storage as a service (STaaS) is a business model in which a large service provider rents space in their storage infrastructure on a subscription basis [7]. Security as a service (SECaaS) is a business model in which a large service provider integrates their security services into a corporate infrastructure on a subscription basis [8].

The STaaS and SECaaS business models can also be used for small organizations where the data is stored using STaaS service and the security is provided by SECaaS service. Personal computer users may have the regular storage units in which the sensitive and personal data are no way connected to the internet.

\section{DROPBOX AND MAILBOX}

Dropbox is a file hosting service operated by Dropbox, Inc. that offers cloud storage, file synchronization, and client software [9]. Dropbox allows users to create a special folder on each of their computers, which Dropbox then synchronises so that it appears to be the same folder (with the same contents) regardless of the computer it is viewed on [10]. Files placed in this folder are also accessible through a web site. The Dropbox service lets users to bring photos, documents and videos and share them easily. Dropbox facility is also available in existing operating systems, but it will be more effective and easy to access in a BUI based operating system.

Mailbox is similar to the Dropbox facility, the difference being that it is not a service provided by external agent rather it is a special folder on the desktop of the operating system. Mailbox is directly connected to the mail accounts of the users. Every now and then when a mail is sent to user's account a notification is shown in the Mailbox. The most attractive feature of Mailbox is that it allows users to integrate all their mail accounts into this special folder.

\section{SPLIT-SCREEN ENVIRONMENT}

The split screen environment that is less commonly used in current operating systems may come to better use in the Browser user interface based operating systems. The user can have one browser screen to open his local system file and another screen to access the internet. Once the user wants to share his file he can share from one screen and view it on 


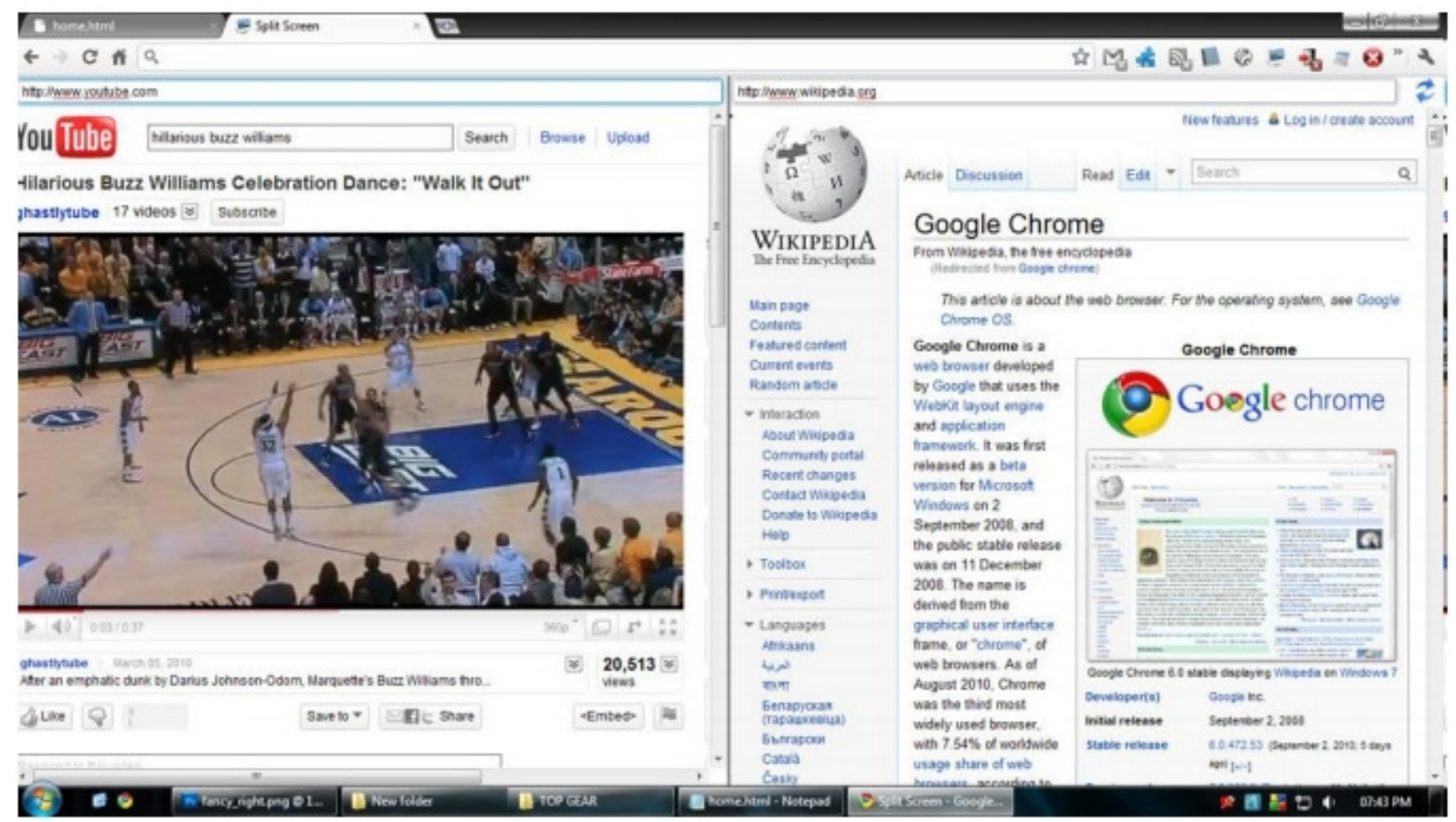

Fig. 1 Split screen environment provided by GOOGLE CHROME OS

the other screen. Such an environment will interest users who like to do multitasking activities.

Apart from the split screen environment, the desktop in a BUI will also have customized icons for accessing the social networking websites similar to the apps in a mobile. This will allow users to access the web with ease. The BUI will also be suited for Private Networks such as VPN's. Example of the split screen environment is shown in fig. 1.

\section{MOZILLA WEBIAN SHELL}

The idea of the Webian Shell project developed by Mozilla is to re-think the computer's interface as something much simpler which treats web applications as first class citizens and does away with all the unnecessary clutter [11]. Shell takes the essential elements of the web browser, desktop environment and window manager and combines them into a single minimalist graphical shell dedicated to using web applications. The vision of Mozilla is to develop an operating interface centred around the browser.

Mozilla has also released an early prototype of such an operating system known as Webian Shell 0.1 [12] shown in fig.2. The Webian Shell is a stripped-down interface that basically shows websites and applications--and not much else. The biggest setback in the Webian Shell is that it is not purely a $\mathrm{BU}$ environment. This is because it offers the option to escape from the browser to the traditional operating system.

Another drawback for the Webian shell is that it can be used with Windows, LinuX and OS X, and while it is far from being, a complete replacement for the operating system and it sits completely on top not allowing the user to interact with the desktop.

\section{GOOGLE CHROME OS}

Chrome OS or Chromium OS is an open-source project that aims to build an operating system that provides a fast, simple, and more secure computing experience for people who spend most of their time on the web. It is a Linux-based operating system designed by Google to work exclusively with web applications. The Google Chrome OS is similar to the home screen that is present in tablets [13].

Early on, Chrome OS was viewed as a competitor to Microsoft Windows. But the two operating systems would not fully overlap in functionality because 


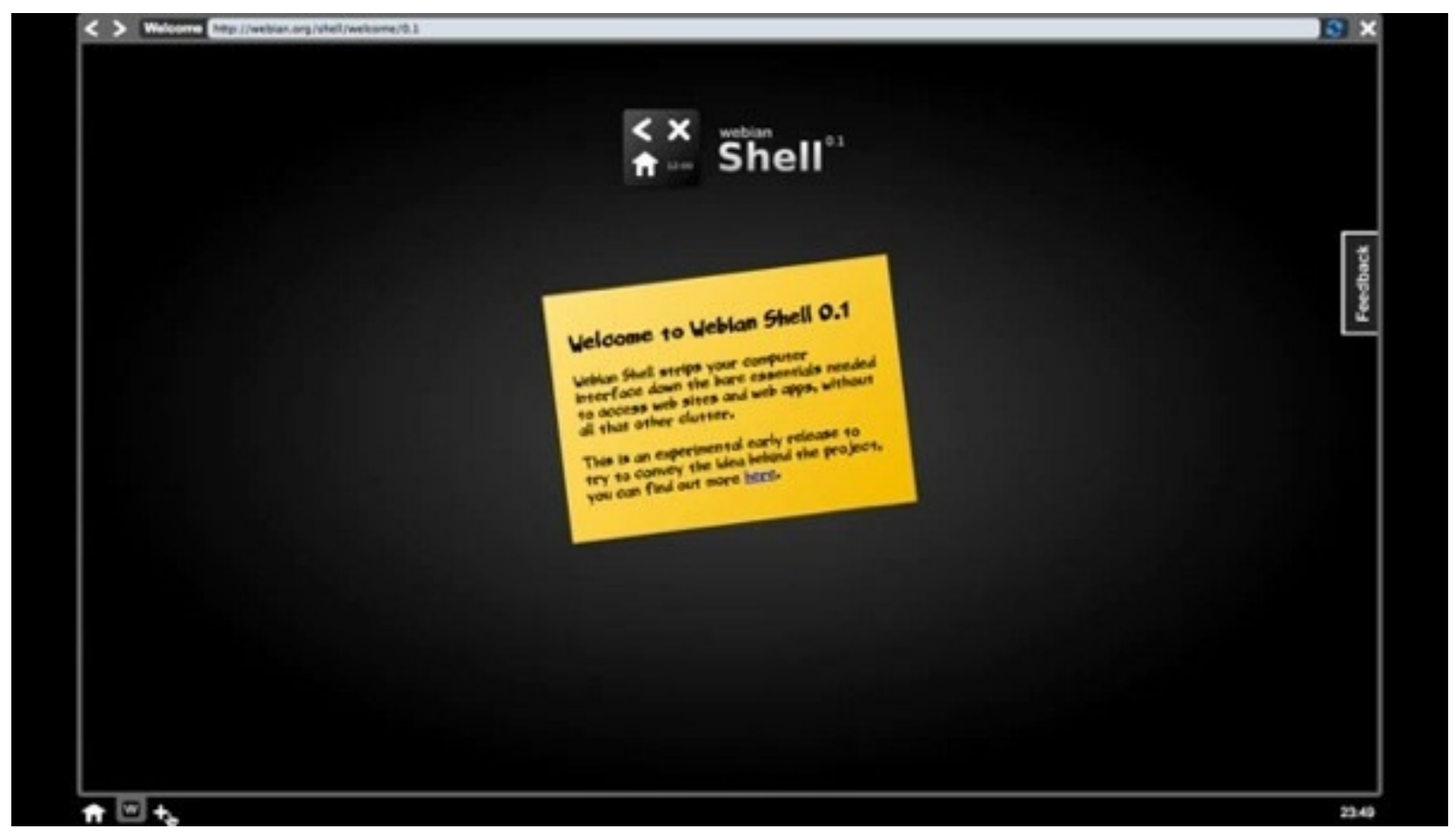

Fig. 2 Mozilla Webian Shell 0.1 prototype

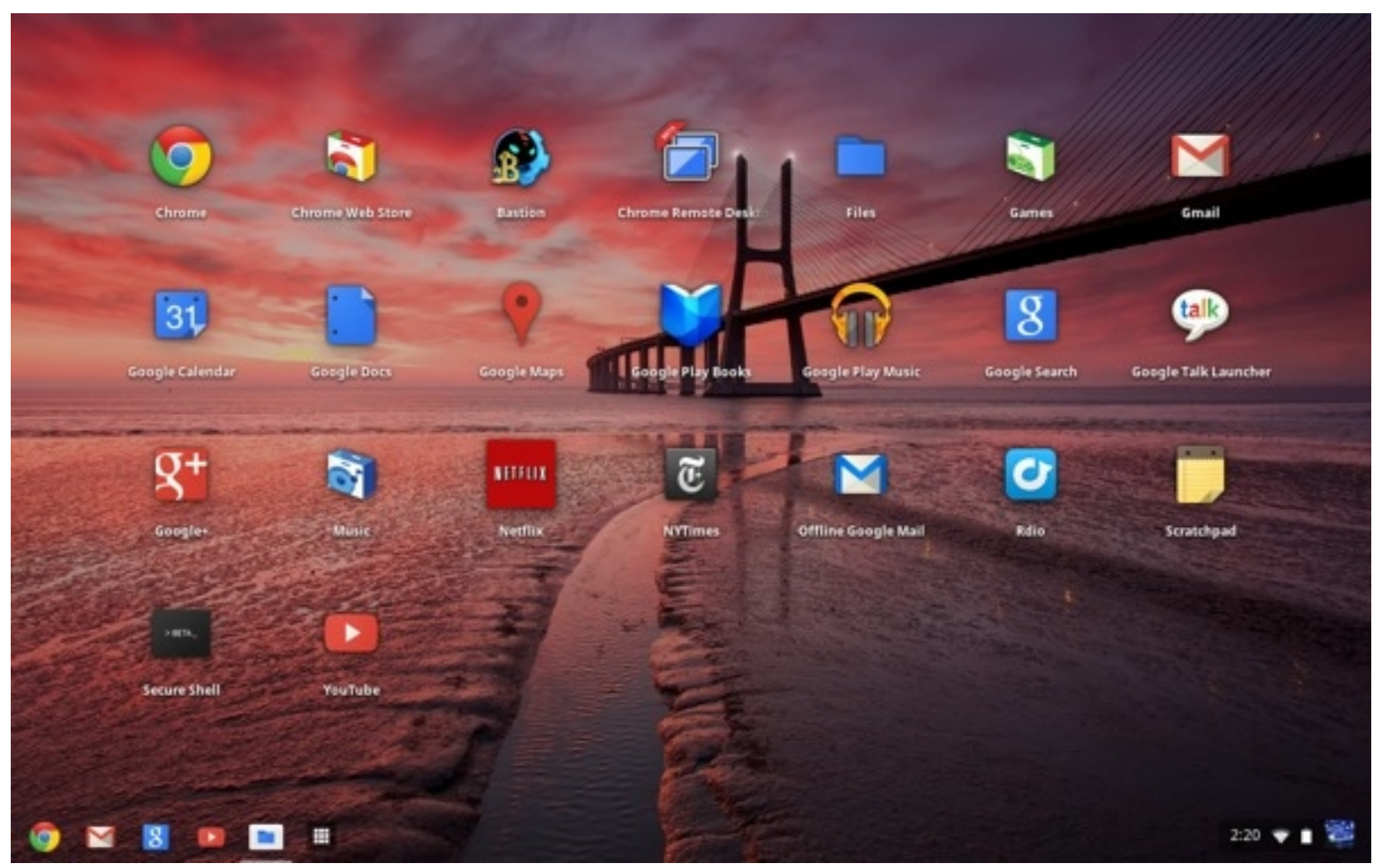

Fig. 3 Desktop of GOOGLE CHROME OS (Version 19) 
Chrome OS hosted is intended for netbooks, which lack the computational power to run a resource-intensive program like Adobe Photoshop.

Chrome OS is not the right setup for everyone. If someone relies on a lot of resource-intensive local programs then Chrome OS might be frustrating to use. While there are plenty of cloud-based apps available for most purposes, the experience using them isn't always as good. The biggest drawback of Google Chrome OS is that it is mainly intended for devices such as notebooks, tablets, etc. Another drawback of the Chrome OS is that it rely primarily on cloud-stored data and does not allow the user to have an option of avoiding clouds.

\section{MICROSOFT'S PAGE VIEW}

Microsoft is working with the World Wide Web Consortium to develop a standard for ActiveX Scripting and the event-driven model. Since IE is integrated with Windows the most famous Operating System developed by Microsoft, VBScript may take centre stage in Windows development as the browser becomes the primary desktop interface. Work is ongoing at Microsoft to completely unify the desktop environment inside the browser and to create a page view. Implementing page view will radically alter the Windows desktop environment. The environment involves Windows Explorer replaced by a browser interface. First and foremost, the concept of a separate browser running on a desktop is eliminated. In fact, the browser will be the desktop. The local files can also be viewed using the browser as if they were web pages [14].

Desktop applications such as Microsoft Office, Microsoft Excel that currently exists can be opened using the browser. In addition to these Microsoft also offers Skydrive which is a cloud computing storage offered by Microsoft. The Skydrive replaces the need for Dropbox. Part of such an environment is achieved in Windows 8 OS.

\section{TECHNICAL ISSUES}

The biggest technical issue that is pertaining to the Browser user interface environment is the potential risk of connecting the local system completely to the internet. The risks include access of data by nonnative users and virus threat from the internet. There are two ways to avoid this security lack. One is the Operating system developer may screen certain areas of the user's system from the internet access. The OS developer must also ensure providing high security from virus for the sensitive data.

Another way is to opt for complete cloud storage. By opting to such a feature the user doesn't need to worry about software installations, updating software, backup facility and also about anti-virus software requirements. This cloud based browser user interface will provide the user with the flexibility to access the stored content from any system or device. The setback of opting for a cloud based $\mathrm{BUI}$ is the price required for storage on clouds. The user is not allowed to access the data on clouds without the internet.

Apart from these issues, another issue that has to be addressed is the extension of browser to behave as the primary point of communication with the system [15]. Current browser application requires a greater change in order to support the features of Browser user interface.

\section{CONCLUSION}

Browser is supposedly the most used application in any system. BUI is a layout that basks on the glory of browsers. Browser user interface will be easy to use and attract users. The notion includes entering url to view local system files, having a share button in text editors, spreadsheets etc, to instantly share the document. BUI eliminates the need to explicitly connect to the internet. All currently existing desktop applications can be opened using the browser. The biggest advantage of a BUI system will the boot time which will be around 7 to 8 seconds against the traditional desktop systems which takes about 45 to 60 seconds to boot.

Ultimately, the user will work with all data in the same way, regardless of the location of the data. For example, local, network, and Internet files will be seamlessly integrated into the browser interface. This represents a significant advance in the user interface because users will no longer have to worry about data location and the technical knowledge necessary to connect to the data.

BUI is a significant development from the Graphical user interface and the Web based user interface. In fact it sandwiches both the Graphical user interface and the Web based user interface in order to 
create a new interface called Browser based user interface.

\section{REFERENCES}

[1] Wilbert O. Galitz "The Essential Guide to User Interface Design: An Introduction to GUI Design Principles and Techniques", $3^{\text {rd }}$ ed.

[2] Ben Shneiderman "Designing the User Interface: Strategies for Effective Human-Computer Interaction", $5^{\text {th }}$ ed.

[3] http://en.wikipedia.org/wiki/User_interface

[4] http://en.wikipedia.org/wiki/Commandline_interface

[5] http://en.wikipedia.org/wiki/Graphical_user_interfac e
[6] http://en.wikipedia.org/wiki/Cloud_computing

[7] http://en.wikipedia.org/wiki/Storage_as_a_service

[8] http://en.wikipedia.org/wiki/Security_as_a_service

[9] http://en.wikipedia.org/wiki/Dropbox_\%28service\% 29

[10] https://www.dropbox.com/about

[11] http://webian.org/shell/

[12] http://www.techspot.com/news/44149-mozilladeveloperlaunches-browser-based-desktopinterface.html

[13] http://www.chromium.org/chromium-os

[14] http://msdn.microsoft.com/enus/library/ms970435.aspx

[15] http://joshondesign.com/2012/07/02/thefutureofdes ktopcomputing 